\title{
La educación básica en México
}

\author{
The basic education in Mexico
}

Nicolás Germán Montiel*

\section{Resumen}

La estructura, organización y fines de la Educación Básica (EB) de México han sufrido importantes cambios en los últimos años, mismos que ha liderado el Presidente de la República Mexicana a través de la Secretaria de Educación Pública (SEP) en conjunción con los gobiernos estatales y el Sindicato Nacional de Trabajadores de la Educación (SNTE), en aras de ofrecer una educación de calidad mediante la cual se forme a las nuevas generaciones de mexicanos(as) por competencias para la vida y el trabajo (saber ser, saber hacer, saber convivir y saber conocer). A través de esta nueva orientación se aspira a que las nuevas generaciones de mexicanos se inserten positivamente en el nuevo entorno socioeconómico y político mundial que se caracteriza por la globalización. Por lo tanto, reflexionar en torno a la descentralización educativa de la EB de México y la forma en que actualmente se encuentra organizada - estructurada, es el objetivo de este trabajo, lo cual permite, deducir los problemas o retos que deben superar los tres órdenes de gobierno y el SNTE si es que realmente se aspira a ofrecer una educación equitativa y de calidad, acorde al nuevo modelo de enseñanza - aprendizaje por competencias

Palabras clave: Educación básica. Rol del docente. Rol del estudiante. Competencias. Evaluación formativa. Centralización y descentralización educativa.

\section{Abstract}

The structure, organization and aims of Basic Education (MB) in Mexico have undergone important changes in recent years, led by the President of the Mexican Republic through the Secretary of Public Education (SEP) in conjunction with governments And the National Union of Education Workers (SNTE), in order to offer a quality education through which the new generations of Mexicans are trained for life and work skills, Knowing how to live and know how to know. Through this new orientation, it is hoped that the new generations of Mexicans will insert themselves positively in the new socioeconomic and political world environment that is characterized by globalization. Therefore, to reflect on the educational decentralization of the MB of Mexico and the way in which it is currently organized - structured, is the objective of this work, which allows, to deduce the problems or challenges that must overcome the three orders of government and the SNTE if it is really aimed at providing an equitable and quality education, according to the new model of teaching - competency learning

Keywords: Basic education. Rol of the teacher. Rol of the student. Competences. Formative evaluation. Centralization and educational descentralization.

Recebido em: 04/03/2017 - Aprovado em: 27/06/2017

http://dx.doi.org/10.5335/rep.v24i2.7415

Investigador independiente y profesor del Plantel Cuauhtémoc de la escuela Preparatoria de la Universidad Autónoma del Estado de México (UAEMéx). Tiene Licenciatura en Antropología Social y Maestría en Práctica docente. E-mail: nico202202@gmail.com 
El siguiente escrito tiene por objetivo principal presentar un panorama general de la forma en que está estructurada - organizada y dirigida la Educación Básica (EB) en México a partir de las reformas implementadas a finales del siglo XX y principios del XXI. Para lograr el objetivo enunciado, se ha estimado pertinente dividir el escrito en tres partes, en la primera se proporciona una breve descripción de los tres acuerdos previos a la Reforma Integral de la Educación Básica en México (RIEB): el Acuerdo Nacional para la Modernización de la Educación Básica en México (ANMEB), el Compromiso social por la Calidad de la Educación y la Alianza por la Calidad de la Educación, posteriormente se define lo que se entiende en México por EB y se proporcionan algunas características de cada nivel educativo que la conforma. En la segunda parte se detalla el papel o funciones de los tres órdenes de gobierno (federal, estatal y municipal) en materia de EB y en la parte final del escrito se establecen algunas consideraciones finales sobre el tema en cuestión.

\section{Acuerdos previos a la Reforma Integral de la Educación Básica (RIEB)}

No es posible comprender la estructura actual del sistema educativo mexicano en lo que a la educación Básica (EB) se refiere, sin tener conocimiento general de tres acontecimientos históricos en los que los actores principales fueron: el Gobierno Federal, los gobiernos estatales y los maestros (representados por el Sindicato Nacional de Trabajadores de la Educación (SNTE). Estos acontecimientos marcan el modelo educativo actual, el cual a su vez determina los planes y programas de estudio, el perfil de egreso, aunado al rol del docente y estudiante.

Todo comenzó con el Acuerdo Nacional para la Modernización de la Educación Básica en México (ANMEB), el cual se llevó acabo en la década de los noventa del siglo veintiuno por el Gobierno Federal, gobiernos estatales y el Sindicato Nacional de Maestros (SNTE). Los tres actores reconocieron la necesidad de realizar cambios a la EB con el fin de elevar su calidad, en aras de formar ciudadanos comprometidos con su superación personal y con el desarrollo económico de su país. Mediante este acuerdo el Gobierno Federal busca corregir y cambiar su política educativa centralista de más de 70 años, es decir, comienza lo que se conoce en México como "descentralización educativa", mediante la cual el Ejecutivo Federal traspasa y el respectivo gobierno estatal recibe, los establecimientos escolares con todos los elementos de carácter técnico y administrativo, derechos y obligaciones, bienes, muebles e inmuebles, con los que la SEP venía prestando en cada estado los servicios de Educación básica, así como los servicios de la educación indígena, educación normal y la educación especial (ANMEB, 1992). 
Ya iniciado el nuevo siglo, concretamente en 2002, el gobierno federal y los gobiernos estatales firmaron el Compromiso Social por la Calidad de la Educación, con el objetivo de ajustar o hacer coincidir el sistema educativo nacional con el contexto económico, político y social en que inicia el nuevo siglo. Se reconoce y adopta el enfoque centrado en el aprendizaje y el enfoque centrado en la enseñanza para que el estudiante aprenda a aprender, aprenda para la vida y a lo largo de la vida, así como a ser ciudadanos que aprecien y practiquen los derechos humanos, la paz, valores y actitudes positivas.

Para completar la transformación de la EB en el año 2008 se firma la Alianza por la Calidad de la Educación entre el Gobierno federal y los maestros de México, representados por el Sindicato Nacional de Trabajadores de la Educación (SNTE). A través de esta alianza se estableció el compromiso de llevar a cabo una reforma curricular orientada al desarrollo de competencias y habilidades, mediante la reforma a los enfoques, asignaturas y contenidos de la Educación Básica. Se determinó que el idioma inglés se impartiera desde el nivel preescolar. Asimismo, se establecieron los compromisos de profesionalizar a los maestros y a las autoridades educativas, y evaluar para mejorar, ya que la evaluación debe servir de estímulo para elevar la calidad de la educación, favorecer la transparencia y la rendición de cuentas, y servir de base para el diseño adecuado de políticas educativas (Acuerdo $592,2011)$.

Todos los acuerdos anteriores se reúnen o sintetizan en la Reforma Integral de la Educación Básica (RIEB) de 2011, a través de la cual el Gobierno Federal como máxima autoridad educativa de México establece la articulación de los tres niveles educativos que la conforman (preescolar, primaria y secundaria) mediante un único modelo educativo por competencias, lo que permite crear un currículum que aborde como un solo trayecto formativo dichos niveles educativos. A partir de esta reforma, se establece que al finalizar la educación básica el estudiante debe poseer un conjunto de competencias que le permitan lograr una vida plena y productiva, coadyuvando en todo momento en el desarrollo integral de su país y de su vida social y personal. Una competencia implica un saber hacer con conocimiento y la valoración de las consecuencias de la aplicación del conocimiento (Acuerdo $592,2011)$. De esta forma un egresado de educación básica debe poseer los conocimientos, habilidades, actitudes y valores que le son necesarios para insertarse en su sociedad como ciudadano activo, con derechos y obligaciones, así como para continuar con su preparación académica en la escuela preparatoria. 


\section{La Educación Básica en México}

En México la educación básica está integrada por los niveles de educación preescolar, primaria y secundaria (LGE, 1993); es decir, comprende los ciclos fundamentales en la instrucción y formación de los educandos, preparatorios para acceder a ciclos medios y superiores o para ingresar al mercado de trabajo y actuar con responsabilidad en su sociedad a partir de que conoce sus derechos y obligaciones. La constitución política de los Estados Unidos Mexicanos (CPEUM) establece que el Estado mexicano de brindar educación básica, laica y gratuita a la población en igualdad de oportunidades y sin discriminación.

Previo a la descripción de la forma en que está estructurado y dirigido cada nivel educativo de la EB pública, se considera pertinente proporcionar algunas características generales que comparten los niveles educativos que la conforman:

a) El director o directora es la primera autoridad en una escuela de preescolar, primaria y/o secundaria, por lo que es responsable del correcto funcionamiento, organización, operación y administración de la escuela y sus anexos.

b) A pesar de que en el artículo $3^{\circ}$ de la Constitución Política de los Estados Unidos Mexicanos se establece que la educación que ofrezca el Estado mexicano a sus ciudadanos debe de ser gratuita; los padres pagan una cuota anual para inscribir a sus hijos(as) a cualquier nivel de educación básica o para reinscribirlos al grado siguiente del nivel educativo que cursan. El dinero lo administra el presidente de una sociedad de padres de familia en cada centro escolar y se utiliza para el pago de conserje (persona que hace la limpieza de las instalaciones educativas), así como para dar mantenimiento al edificio escolar. Los padres también deben comprar el uniforme de gala y deportivo que el alumno(a) portará durante la semana escolar, comprar la lista de útiles escolares (cuadernos, lápices, lapiceros, colores, juego geométrico, hojas blancas, etc.) al inicio y durante el año escolar, así como, sufragar todos los gastos que se deriven de su estancia en la escuela. Lo gratuito de la EB en México parece que se encuentra en tela de juicio, porque aunado a lo anterior, en el nivel preescolar y escuela primaria son los padres de familia quienes pagan al docente de la materia de inglés y computación. Solo en secundaria el pago es realizado por el Gobierno federal a través del gobierno estatal.

c) La semana escolar consta de cinco días, los alumnos y alumnas portan uniforme de gala cuatro días, y el quinto día deben portar su uniforme deportivo.

d) A los niños de primaria y secundaria se les proporcionan libros de texto gratuitos. 
e) La SEP establece los planes y programas de estudio para la educación preescolar, primaria y secundaria, su observancia es de carácter nacional y general para todos los establecimientos, públicos y privados.

f) Los docentes de Educación Básica son egresados(as) de escuelas normales del estado y de universidades públicas y/o privadas en que se ofrece la licenciatura en Educación o Pedagogía.

g) Los programas de estudio, la planeación didáctica y los propósitos de aprendizaje están en concordancia con el Modelo educativo por competencias que el gobierno adoptó para todo el sistema educativo nacional a partir de la Reforma Integral de la Educación Básica (RIEB).

A continuación se proporciona algunas características de los niveles educativos que conforman la EB en México en la modalidad o servicio general, ya que existe la modalidad comunitaria (localidades con menos de 500 habitantes) e indígena, modalidades que no se abordan en este escrito..

\section{El nivel preescolar}

En México la educación preescolar que se ofrece mediante el servicio general es el más extendido, ya que $67 \%$ de las poco más de 90 mil escuelas que hay en el país, pertenecen a esta modalidad (INEE, 2012). En el año 2004 se realizó una reforma a la Ley General de educación de 1993, para establecer que es obligatorio que los niños y niñas de México cursen el preescolar de los tres a los cinco años. Tal como lo establece la RIEB, al finalizar este nivel educativo los niños y niñas deben haber aprendido conocimientos, habilidades y actitudes que les permitan continuar con sus estudios en la escuela primaria, y crecer como personas (individual y socialmente).

Entre los propósitos fundamentales de aprendizaje de la educación preescolar pueden citarse los siguientes: que el niño o niña desarrolle un sentido positivo de sí mismo, exprese sus sentimientos, empiece a regular sus emociones, muestre disposición para aprender, trabajar en equipo, resolver conflictos a través del diálogo, amplíen su vocabulario, conozcan las principales funciones del lenguaje escrito, reconozcan que las personas tenemos rasgos culturales distintos, establezcan relaciones de correspondencia, cantidad y ubicación entre objetos, para estimar y contar, para reconocer atributos y comparar, desarrollen la capacidad para resolver problemas de manera creativa mediante situaciones de juego que impliquen la reflexión y la explicación; se interesen en la observación de fenómenos naturales, y participen en situaciones de experimentación que les permitan preguntar predecir, comparar, registrar, elaborar, etc. (Acuerdo 348, 2004). Los anteriores propósitos 
deben lograrse por el profesor(a) en los campos formativos que conforman el nivel de preescolar: desarrollo personal y social, lenguaje y comunicación, pensamiento matemático, exploración y conocimiento del mundo, expresión y apreciación artística, y desarrollo físico y salud.

Respecto a la evaluación de los aprendizajes, está tendrá un carácter eminentemente formativo, porque se utilizará solo como un medio para la mejora continua del alumno(a) y no para determinar si acredita o no acredita un año escolar. De esta forma no se proporcionará a los padres una boleta de aprobación de cada grado cursado por el niño o niña, solo se les entrega una constancia correspondiente de que el niño(a) ha cursado dicho grado de preescolar (Acuerdo 348).

\section{La escuela primaria}

México cuenta con casi 100 mil escuelas de educación primaria, la mayoría de ellas son de tipo general (78\%), una de cada 10 son indígenas y 11 comunitarias, nueve de cada 10 primarias son de financiamiento público. En cualquiera de sus modalidades, la educación primaria es obligatoria y se cursa en seis grados, ya que es previa e indispensable para cursar la educación secundaria. Al primer grado acceden normalmente los niños de seis años cumplidos que acaban de finalizar su educación preescolar, y concluyen el sexto grado, alumnos que por lo regular tienen 11 años, aunque son aceptados como alumnos hasta los 15 años, dado que pueden haber ingresado a primaria con más de seis años cumplidos y haber tenido bajas temporales o repetición de grados en el trayecto.

\section{Propósitos de aprendizaje de la educación primaria}

A través del Acuerdo 181 de1993, el Gobierno Federal por medio de la SEP establece que todos los niños y niñas que cursen la educación primaria:

1. Desarrollen sus habilidades intelectuales y los hábitos que les permitan aprender permanentemente y con independencia, así como actuar con eficacia e iniciativa en las cuestiones prácticas de la vida cotidiana, tales como la lectura y la escritura, la expresión oral, la búsqueda y selección de información, y la aplicación de las matemáticas a la realidad.

2. Adquieran los conocimientos fundamentales para comprender los fenómenos naturales, en particular los que se relacionan con la preservación de la salud, con la protección del ambiente y con el uso racional de los recursos naturales, así como aquellos que proporcionan una visión organizada de la historia y la geografía de México. 
3. Se formen éticamente mediante el conocimiento de sus derechos y deberes, y la práctica de valores en su vida personal, en sus relaciones con los demás y como integrantes de la comunidad nacional.

4. Desarrollen actitudes propicias para el aprecio y disfrute de las artes y del ejercicio físico y deportivo.

Para lograr los anteriores propósitos, se ha establecido que en primero y segundo grados los niños y niñas cursen las siguientes asignaturas: Español, Matemáticas, Conocimiento del Medio (trabajo integrado de Ciencias Naturales, Historia, Geografía y Educación Cívica), Educación Artística y Educación Física. De tercer a sexto grado se imparten: Español, Matemáticas, Ciencias Naturales, Historia, Geografía, Educación Cívica, Educación Artística y Educación Física.

\section{Los criterios de promoción o acreditación son:}

La escala oficial de calificaciones es numérica del 5 al 10, siendo 6.0 la calificación mínima aprobatoria.

\section{a) Para el primer grado}

Primero y segundo grados de primaria, son considerados como partes de un ciclo. Muchos alumnos que no aprenden a leer y escribir en primero, lo hacen sin mayores problemas en segundo. Por lo tanto, el alumno que haya asistido regularmente a clases debe ser PROMOVIDO a segundo, a menos que el maestro detecte problemas serios de aprendizaje. Se recomienda que al decidir reprobar a un alumno de primer grado de primaria se tomen en cuenta las opiniones del padre de familia o tutor y de las autoridades de la escuela.

b) Segundo a sexto grado

Un alumno es PROMOVIDO, cuando obtiene Calificación Final aprobatoria en Español y Matemáticas, y si además, su Promedio General Anual es mayor o igual a 6.0.

De igual forma, deberá promoverse al alumno que obtenga calificaciones finales menores que 6.0 en algunas de las asignaturas que no sean Español ni Matemáticas, y su Promedio General Anual sea de 6.0 o más.

NO PROMOVIDO: El alumno no será promovido si reprueba Español o Matemáticas, o si aprueba esas dos asignaturas, pero su Promedio General Anual es menor de 6.0 
Los documentos oficiales que se utilizan para dar validez al proceso de certificación son los siguientes: A) Certificado de Terminación de Estudios, B) Resolución de Revalidación de Estudios (únicamente para sexto grado), C). Certificado de Estudios, D) Boleta de Evaluación de sexto grado y E) Relación de Folios de Certificados de Terminación de Estudios (Acuerdo 181, 1993).

\section{La escuela secundaria}

La secundaria se proporciona en tres años a quienes hayan concluido la educación primaria, generalmente está dirigida a la población de 12 a 16 años de edad. De acuerdo con el INEE (2012), en México existen poco más de 36500 escuelas secundarias, en las que estudian casi 6.2 millones de jóvenes. La educación secundaria se imparte en cinco tipos: telesecundaria, a la que corresponden la mitad de las escuelas; general, con $31 \%$ de los planteles; técnica (13\%); comunitaria (6\%), y las secundarias para trabajadores, con $1 \%$. La gran mayoría de las escuelas secundarias (88\%) es de financiamiento público. A partir de 1993 la educación secundaria en México se define como el último tramo de la enseñanza básica obligatoria (Zorrilla, 2004). Lamentablemente, si bien se ha ampliado su cobertura y la asistencia de la población; la reprobación y la deserción no permiten que la eficiencia terminal en este nivel este mejorando sustancialmente.

El propósito esencial del plan de estudios de la secundaria es asegurar que todos los adolescentes adquieran las herramientas para aprender a lo largo de toda su vida, porque en la actualidad, las necesidades de aprendizaje se relacionan con la capacidad de reflexión y el análisis crítico; el ejercicio de los derechos civiles y democráticos; la producción y el intercambio de conocimientos a través de diversos medios; el cuidado de la salud y del ambiente, así como con la participación en un mundo laboral cada vez más versátil (Acuerdo 384, 2006).

De esta forma, las prioridades del plan de estudios de la secundaria son:

1. Asegurar que los estudiantes profundicen y ejerciten su competencia para utilizar el español en forma oral y escrita; desarrollar las capacidades de expresar ideas y opiniones con precisión y claridad; entender, valorar y seleccionar material de lectura, en sus diferentes funciones informativas, prácticas y literarias. A las actividades relacionadas directamente con el lenguaje, se dedican cinco horas de clase a la semana y se promueve que las diversas competencias lingüísticas se practiquen sistemáticamente en las demás asignaturas.

2. Ampliar y consolidar los conocimientos y habilidades matemáticas, las capacidades para aplicar la aritmética, el álgebra y la geometría en el plan- 
teamiento y la resolución de problemas de la actividad cotidiana, y para entender y organizar información cuantitativa. A la asignatura de Matemáticas se destinan de manera específica cinco horas semanales y en las diversas asignaturas se propicia la aplicación de las formas de razonamiento y de los recursos de las Matemáticas.

3. Fortalecer la formación científica de los estudiantes y superar los problemas de aprendizaje que se presentan en este campo. En el primer año de la secundaria, existe un curso de Introducción a la Física y a la Química, cuyo propósito es facilitar la transición del estudio por área que se realiza en la educación primaria al estudio por disciplinas en la secundaria. En el segundo y tercer grados, la Física, la Química y la Biología se estudian por separado como asignaturas específicas. El enfoque de estos cursos es establecer una vinculación continua entre las ciencias y los fenómenos del entorno natural que tienen mayor importancia social y personal, como son la protección de los recursos naturales y del ambiente, la preservación de la salud y la comprensión de los procesos de cambio que caracterizan a la adolescencia.

4. Profundizar y sistematizar la formación de los estudiantes en Historia, Geografía y Civismo, con el objetivo de que adquieran elementos para entender los procesos de desarrollo de las culturas humanas; desarrollar en los educandos una visión general del mundo contemporáneo y de la interdependencia creciente entre sus partes; inducir su participación en relaciones sociales regidas por los valores de la legalidad, el respeto a los derechos, la responsabilidad personal y el aprecio y defensa de la soberanía nacional.

5. El aprendizaje de una lengua extranjera (inglés o francés), en particular sus aspectos de uso más frecuentes en la comunicación.

El plan de estudios destina espacios a actividades que tienen un papel fundamental en la formación integral del estudiante: la expresión y apreciación artística, la educación física y la educación tecnológica.

\section{Criterio de promoción 0 acreditación}

La escala oficial de calificaciones para cada asignatura es numérica del 5 al 10, siendo 6.0 la calificación mínima aprobatoria. Los documentos oficiales que se utilizan para dar validez al proceso de certificación son los siguientes: a) Certificado de Terminación de Estudios, b) Certificación de Estudios, c) Boleta de Evaluación, d) Relación de Folios de Certificados de Terminación de Estudios, e) Resolución de 
Equivalencia o Revalidación de Estudios y f) Informe de Calificaciones de Estudios Parciales (Acuerdo 384, 2006).

\section{El papel del Gobierno federal, estados y municipios en la Educación Básica}

Esta parte del escrito es pertinente comenzarla con la siguiente pregunta: ¿Quién dirige la educación básica en México? Porque, a partir de la descentralización educativa, parece que los tres órdenes de gobierno comparten el presente y futuro de la EB en México.

Una historia oscura...

Antes, durante y al final de la Revolución Mexicana e inicio de los gobiernos elegidos por vía democrática, se visualiza a la educación como el medio para la unificación del país, detonar su desarrollo económico, lo que permitiría disminuir hasta erradicar las desigualdades económicas y culturales entre los grupos más atrasados del país (campesinos e indígenas) para con los mestizos y criollos. Para lograr este objetivo, se crea la Secretaria de Educación Pública (SEP) en 1921, lamentablemente este periodo de federalización educativa se alejó de su vía democrática o buenas intenciones, porque más que distribuir las responsabilidades educativas entre municipios, estados y gobierno federal, fue este último el que terminó quedándose con la responsabilidad casi absoluta de la educación; creando un centralismo educativo que duró casi siete décadas, pues la SEP se burocratizó, lo cual se manifestó en la lentitud en los tramites, multiplicación de líneas de autoridad entre funcionarios a nivel nacional, estatal y local, duplicación de esfuerzos, confusión de mandos, etc. Esta situación llevó a que la responsabilidad de los municipios se diluyera en el sistema estatal y este, quedó cada vez más supeditado al gobierno federal, en pocas palabras, prácticamente los municipios y estados dejaron la responsabilidad educativa en manos del gobierno federal (RODRÍGUEZ REYES; MEZA MEDINA, s.f.). Tal como citó en la primera parte de este escrito, el Gobierno Federal junto con los gobiernos estatales y maestros (representados por el SNTE) decidió realizar lo que se conoce como Descentralización educativa. La cual se caracteriza principalmente por lo siguiente: el Gobierno Federal comenzó a delegar funciones a los gobiernos estatales y municipales en materia educativa.

Para determinar con más claridad qué funciones respecto a la educación conservó el gobierno federal y cuáles cedió a los estados, y municipios. A continuación se describen brevemente las funciones que corresponden para con la EB a cada orden de gobierno: 
Responsabilidades del gobierno federal para con la educación básica:

El gobierno federal, por conducto de la Secretaria de Educación Pública, detenta la autoridad normativa, técnica y pedagógica para el funcionamiento de la educación básica. En el artículo 12 de la LGE de 1993 se establece que la autoridad educativa federal tiene entre otras atribuciones las siguientes con respecto a la educación básica:

- Determinar los planes y programas de estudio de la educación preescolar, la primaria y la secundaria; es decir, para toda la educación básica.

- Realizar para toda la República la planeación global del sistema escolar de educación básica, evaluar a este y fijar los lineamientos generales de la evaluación que las autoridades educativas locales deban realizar.

- Establecer el calendario escolar aplicable en toda la República para cada ciclo lectivo de la educación preescolar, la primaria, la secundaria

- Determinar contenidos escolares de la educación básica.

- Elaborar y mantener actualizados los libros de texto gratuitos para la educación primaria y secundaria

- Autorizar el uso de libros de texto para la educación primaria y la secundaria.

- Regular un sistema nacional de formación, actualización, capacitación y superación profesional para maestros de educación básica.

- Fijar los requisitos pedagógicos de los planes y programas de educación inicial y preescolar que, en su caso, formulen los particulares.

- Propiciar el desarrollo educativo armónico entre las entidades federativas, concertando con éstas las acciones necesarias para reducir y superar disparidades.

- Dar atención prioritaria a aquellas regiones con importantes rezagos educativos.

- Establecer procedimientos de evaluación del sistema educativo nacional, promoverá los servicios educativos que faciliten a los educadores su formación y constante perfeccionamiento, y fomentará permanentemente la investigación que permita la innovación educativa.

Con lo escrito en las líneas anteriores se puede deducir o inferir que el gobierno federal a través de la SEP sigue manteniendo el control sobre los objetivos nacionales y lineamientos generales de la política educativa de México; sobre los contenidos, planes y/o programas educativos, determinado el modelo educativo a seguir en los niveles de educación básica, así como la elaboración y/o reedición de los libros de texto para la escuela primaria y secundaria. Por lo tanto, apegándose a estos atributos en materia de educación, el Gobierno Federal determinó realizar una Reforma Educativa de la EB, centrada en la adopción del modelo educativo 
por competencias que responda a las necesidades de desarrollo de México en el siglo XXI, reforma que exige la formación continua y evaluación permanente de los docentes para acreditar su idoneidad (RUÍZ CUÉLLAR, 2012).

Responsabilidades de las autoridades locales (gobierno estatal y municipal) para con la Educación Básica:

De acuerdo con la LGE de 1993, en su artículo 13, corresponden de manera exclusiva a las autoridades educativas locales, en sus respectivas competencias, las atribuciones siguientes:

I. Prestar los servicios de educación inicial, educación básica incluyendo la indígena, especial, así como la normal y demás para la formación de maestros;

II. Proponer a la Secretaría los contenidos regionales que hayan de incluirse en los planes y programas de estudio para la educación preescolar, la primaria, la secundaria, la normal y demás para la formación de maestros de educación básica;

III. Ajustar, en su caso, el calendario escolar para cada ciclo lectivo de la educación preescolar, la primaria, la secundaria, la normal y demás para la formación de maestros de educación básica, con respeto al calendario fijado por la Secretaría;

IV. Prestar los servicios de formación, actualización, capacitación y superación profesional para los maestros de educación básica, de conformidad con las disposiciones generales que la Secretaría determine;

V. Revalidar y otorgar equivalencias de estudios de la educación preescolar, la primaria, la secundaria, la normal y demás para la formación de maestros de educación básica, de acuerdo con los orientaciones generales que la Secretaría expida;

VI. Otorgar, negar y revocar autorización a los particulares para impartir la educación preescolar, la primaria, la secundaria, la normal y demás para la formación de maestros de educación básica, y

VII.Las demás que con tal carácter establezcan esta Ley y otras disposiciones aplicables.

\section{Consideraciones finales}

El análisis realizado nos permite reconocer el verticalismo que caracteriza la toma de decisiones en materia de EB, la cual es considerada un aspecto nodal para el crecimiento personal y social del alumno, así como para el desarrollo eco- 
nómico del país. Tal parece que la descentralización educativa en México sigue siendo un mito más que una realidad, porque el Gobierno Federal continua siendo el responsable directo a través de la SEP de la creación o modificación del modelo educativo, planes y programas de estudio; no permitiendo a los gobiernos estatales y municipales adquirir la responsabilidad total de estos aspectos en sus respectivos estados y municipios. Por otro lado, la EB sigue presentando retos importantes como el número de estudiantes por aula que se tiene en preescolar, primaria y secundaria. Por ejemplo, a nivel primaria un solo profesor debe atender a 30 o 40 alumnos durante cada jornada escolar; en secundaria, el panorama es todavía más grave, porque un solo maestro debe atender a 40 o 50 alumnos(as) por grupo, sin olvidar que le son asignados entre 6 y 10 grupos. Esta situación se agudiza debido a que la mayoría de los docentes no cuentan con conocimientos psicopedagógicos y/o profesionales para atender a ese número de estudiantes, cuestión que favorece su refugio en actitudes autoritarias y represoras. Si a esta situación se agrega que la mayoría de los profesores en secundaria no cuentan con la planta docente, lo cual les proporcionaría tiempo y espacio en la escuela para trabajar de forma colegiada en proyectos relacionados con el apoyo a alumnos(as) en situación de riesgo, proyectos o trabajos escolares interdisciplinares y multidisciplinares, etc. tal como lo exige el modelo educativo por competencias.

Por otro lado, es un hecho que la EB en México ni es totalmente gratuita ni ofrece la calidad que los discursos gubernamentales y/o políticos enfatizan, porque los padres deben pagar cada hora clase de inglés y/o computación que sus hijos reciben en el preescolar y primaria. Aunado a lo anterior, padres de familia, hijos, políticos y gobiernos, siguen más preocupados por la calificación que se obtiene al final de cada asignatura del grado escolar que cursaron, que en los aprendizajes alcanzados y/o grado de desarrollo de las competencias que forman parte del perfil de egreso de cada nivel educativo de la EB. Es cierto que todo modelo educativo requiere de tiempo para la evaluación de su impacto en la formación de ciudadanos y profesionistas de una sociedad; sin embargo, mientras padres de familia, docentes, directivos, autoridades municipales, estatales y Gobierno Federal no compartan las mismas ideas en torno a los fines y funciones de la educación, y tampoco asuman el papel que les corresponde en la formación de las nuevas generaciones de mexicanos, nunca se obtendrán los resultados que se esperan. 


\section{Referencias}

ACUERDO número 181, por el que se establecen el plan y los programas de estudio para la educación primaria (27 de agosto de 1993). Diario oficial de la federación.

ACUERDO 348 por el que se determina el Programa de Educación Preescolar (27 de octubre de 2004). Diario oficial de la federación.

ACUERDO número 384 por el que se establece el nuevo Plan y Programas de Estudio para Educación Secundaria (26 de mayo de 2006). Diario oficial de la federación.

ACUERDO número 592 por el que se establece la articulación de la educación básica (viernes 19 de agosto de 2011). Diario oficial de la federación.

ANMEB. Acuerdo Nacional para la Modernización de la Educación Básica. 1992.

INSTITUTO NACIONAL PARA LA EVALUACIÓN DE LA EDUCACIÓN. La educación en México: Estado actual y consideraciones sobre su evaluación. 2012.

MÉXICO. Secretaria de Educación Pública. Decreto por el que se reforman diversas disposiciones de la Ley General de Educación, en materia de educación preescolar (10 de diciembre de 2004).

MÉXICO. Secretaria de Educación Pública. Ley General de Educación (martes 13 de julio de 1993).

RODRÍGUEZ REYES, C. S.; MEZA MEDINA, G. (s.f.). Federalización educativa en México... ¿y la educación? XI Congreso Nacional de Investigación Educativa, 13. Política y Gestión / Ponencia.

RUÍZ CUÉLLAR, G. La Reforma Integral de la Educación Básica en México (RIEB) en la educación primaria: desafíos para la formación docente. Revista Electrónica Interuniversitaria de Formación del Profesorado, v. 15, n. 1, p. 51-60, 2012.

ZORRILLA, M. La educación secundaria en México: Al filo de su reforma. Revista Electrónica Iberoamericana sobre Calidad, Eficacia y Cambio en Educación, v. 2, n. 4, 2004.

ZORRILLA, M.; BARBA, B. (s.f.). Reforma educativa en México. Descentralización y nuevos actores. 